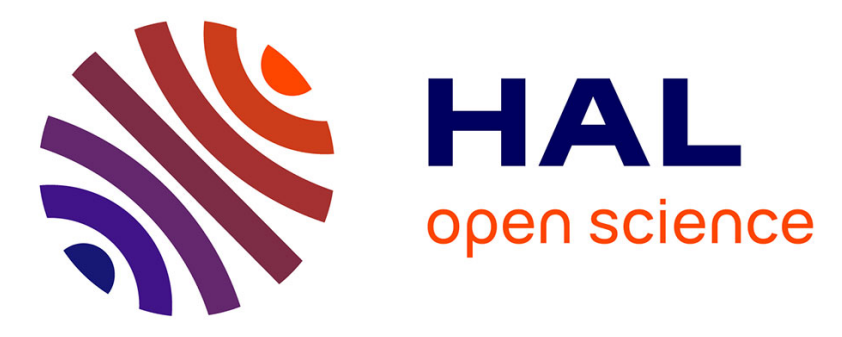

\title{
Direct Observation of Slow Light in the Noise Spectrum of a Laser
}

\author{
Abdelkrim El Amili, B.-X. Miranda, Fabienne Goldfarb, Ghaya Baili, \\ Grégoire Beaudoin, Isabelle Sagnes, Fabien Bretenaker, Mehdi Alouini
}

\section{- To cite this version:}

Abdelkrim El Amili, B.-X. Miranda, Fabienne Goldfarb, Ghaya Baili, Grégoire Beaudoin, et al.. Direct Observation of Slow Light in the Noise Spectrum of a Laser. CLEO:2011 Laser Applications to Photonic Application, May 2011, Baltimore, United States. pp.JThB65, 10.1364/CLEO_AT.2011.JThB65 . hal-01044918

\author{
HAL Id: hal-01044918 \\ https://hal.science/hal-01044918
}

Submitted on 24 Jul 2014

HAL is a multi-disciplinary open access archive for the deposit and dissemination of scientific research documents, whether they are published or not. The documents may come from teaching and research institutions in France or abroad, or from public or private research centers.
L'archive ouverte pluridisciplinaire HAL, est destinée au dépôt et à la diffusion de documents scientifiques de niveau recherche, publiés ou non, émanant des établissements d'enseignement et de recherche français ou étrangers, des laboratoires publics ou privés. 


\title{
Direct Observation of Slow Light in the Noise Spectrum of a Laser
}

\author{
A. El Amili ${ }^{1}$, B.-X. Miranda ${ }^{1,2}$, F. Goldfarb ${ }^{1}$, G. Baili ${ }^{3}$, G. Beaudoin ${ }^{4}$, I. Sagnes ${ }^{4}$, \\ F. Bretenaker ${ }^{1}$ and M. Alouini ${ }^{2,3}$ \\ ${ }^{1}$ Laboratoire Aimé Cotton, CNRS-Université Paris Sud 11, 91405 Orsay Cedex, France \\ ${ }^{2}$ Institut de Physique de Rennes, CNRS-Université de Rennes I, 35042 Rennes Cedex, France \\ ${ }^{3}$ Thales Research and Technology, Campus Polytechnique, 91127 Palaiseau Cedex, France \\ ${ }^{4}$ Laboratoire de Photonique et Nanostructures, CNRS, Route de Nozay, 91460 Marcoussis, France \\ karim.el-amili@u-psud.fr \\ fabien.bretenaker@u-psud.fr
}

\begin{abstract}
The role of coherent population oscillations is evidenced in the noise spectrum of an ultra-low noise laser. The coherent population oscillations manifest themselves through their associated dispersion probed by the non-lasing side modes.
\end{abstract}

(C) 2010 Optical Society of America

OCIS codes: $140.5960,190.4380,270.2500$.

\section{Introduction}

Slow and fast light (SFL) have been the subject of considerable research efforts. To control the group velocity of light, various approaches have been proposed and demonstrated, such as, e. g., electromagnetically induced transparency [1, 2], coherent population oscillations (CPO) [3], and stimulated Brillouin scattering [4]. CPO, an ubiquitous mechanism inducing SFL, is present in any active medium provided that a strong optical beam saturates this medium. Thus, CPO must be present in any single frequency laser since the oscillating beam acts as a strong pump which, by definition, saturates the active medium. This effect could be observed using an external probe whose angular frequency is detuned with respect to the oscillating mode, by less than the inverse of the population inversion lifetime $1 / \tau_{c}$. Consequently, this effect should be also visible in the laser excess noise, using the spontaneous emission present in the non-lasing side longitudinal modes of a single-frequency laser as probe of the CPO effect. In semiconductor lasers $\tau_{\mathrm{c}}$ is in the ns range, and then the $\mathrm{CPO}$ effects are efficient at offset frequencies below a few $\mathrm{GHz}$ from the lasing mode [5]. However, class-A vertical external cavity surface emitting semiconductor lasers (VECSELs) [6] recently developed for their low noise characteristics exhibit i) single-frequency operation, ii) ultra-narrow linewidth, iii) shot-noise limited intensity noise, and iv) a FSR in the GHz range. All these characteristics make them perfectly suited for the observation of CPO induced SFL in their noise spectrum.

\section{Experimental setup and results}

The laser used in our experiment is a VECSEL which operates at $\sim 1 \mu \mathrm{m}$. The $1 / 2$-VCSEL gain chip is a multi-layered stack of semiconductors materials. Gain is produced by six InGaAs/GaAsP strained quantum wells grown on a high reflectivity Bragg mirror. The top of the gain structure is covered by an anti-reflection coating. The output mirror is placed at $L \lesssim 10 \mathrm{~cm}$ from the gain structure. In these conditions, $1 / 2 \pi \tau_{\mathrm{c}}$ is not negligible compared with the FSR $(\Delta \gtrsim 1.5 \mathrm{GHz})$. The laser is optically pumped at $808 \mathrm{~nm}$. A thick glass etalon is inserted inside the cavity to make the laser single mode. The noise spectrum is measured using a wide bandwidth photodiode and a low noise radiofrequency amplifier. We focus on the excess noise due to the beat notes between the laser line and the spontaneous emission noise at neighboring longitudinal mode frequencies [7,8]. At the $p^{\text {th }}$ FSR frequency $p \Delta$, the noise spectrum is thus the sum of two Lorentzian peaks due to the beat notes of the lasing mode with the corresponding sidebands $\left(p^{\text {th }}\right.$ and $-p^{\text {th }}$ modes). When the pumping rate is increased, we found experimentally that the excess noise consists of two peaks separated by $\delta f=f_{p}-f_{-p} \sim 100 \mathrm{kHz}$ (inset of Fig. 1). This frequency shift is given by

$$
\delta f \approx v_{0} \frac{L_{m}}{L+n_{0} L_{m}}\left(\delta n_{p}+\delta n_{-p}\right)
$$




\section{JThB65.pdf}
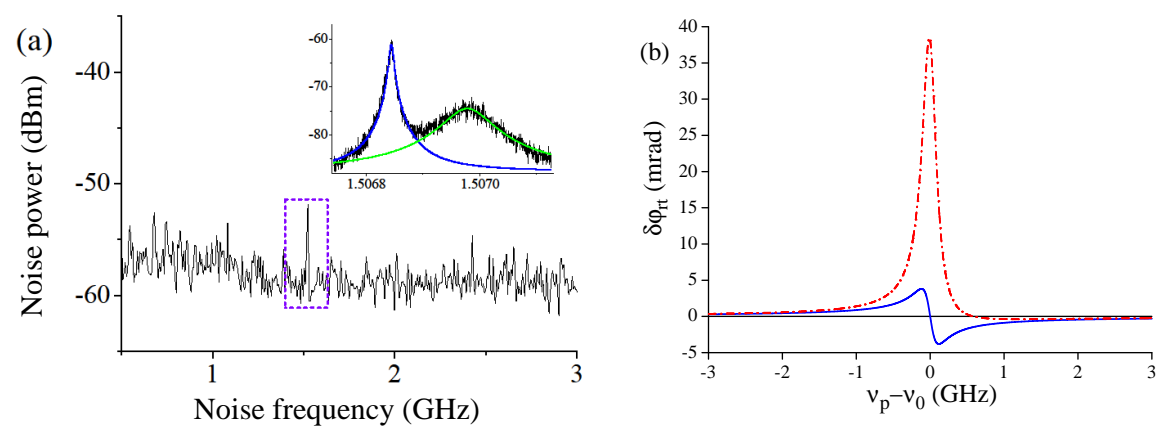

Fig. 1. (a) Typical laser intensity noise spectrum. For a cavity length $L \approx 10 \mathrm{~cm}$, the beat note frequency appear at the first harmonic of the resonator FSR $\Delta \approx 1.5 \mathrm{GHz}$. The inset is a zoom of the excess noise in the region around $\Delta$. (b) Round-trip phase modification experienced by the side modes for $\alpha=0$ (full line) and $\alpha=5$ (dotted-dashed line).

where $n_{0}$ is the bulk refractive index of the semiconductor structure and $L_{m}$ is the length of the gain medium. $\delta n_{ \pm p}$ are the modifications of the refractive index of the structure experienced by the $\pm p$ side modes and induced by the dispersion associated with the CPO effect. In a semiconductor active medium, thanks to the Bogatov effect [10], the dispersion is not an odd function of the frequency detuning with respect to $v_{0}$. Thus, $\delta n_{p} \neq-\delta n_{-p}$ and the two beat note frequencies $f_{p}$ and $f_{-p}$ corresponding to the $p$ and $-p$ modes occur at slightly different frequencies, as evidenced by the double peak of Fig. 1(a). This CPO induced index modification can be derived from the gain medium rate equation including the phase-intensity coupling coefficient $\alpha$ (Henry's factor) that is responsible for the Bogatov effect [11]. This CPO effect is also responsible for the modification of the refractive index seen by the side modes which modifies the round-trip phase accumulated by each side mode Fig. 1(b). Finally, notice also that since the cavity FSR $\Delta$ is sufficiently large that we are probe the wings of the dispersion profile of Fig. 1(b), i. e., in the slow light regime.

\section{Conclusion}

In conclusion, we experimentally evidenced the existence of intracavity slow light effects in a laser induced by the CPO mechanism. These effects are probed by the laser spontaneous emission noise present in the non lasing modes. We have shown that this noise is a very efficient probe to explore the intracavity CPO effects.

The authors acknowledge partial support from the Agence Nationale de la Recherche, the Triangle de la Physique, and the Région Bretagne.

\section{References}

1. S. E. Harris, J. E. Field, and A. Imamoğlu, Phys. Rev. Lett. 64, 1107 (1990).

2. L. V. Hau, S. E. Harris, Z. Dutton, and T. Behroozi, Nature 397, 594 (1999).

3. M. S. Bigelow, N. N. Lepeshkin, and R. W. Boyd, Phys. Rev. Lett. 90, 113903 (2003).

4. L. Thevenaz, Nature Photonics 2, 474 (2008).

5. G. P. Agrawal and N. K. Dutta, Semiconductor Lasers, 2nd edition (Springer, Berlin, 1993).

6. G. Baili et al., Opt. Lett. 31, 62-64 (2006).

7. M. P. van Exter, R. F. M. Hendriks, J. P. Woerdman, and C. J. van der Poel, Opt. Comm. 110, 137 (1994).

8. G. Baili et al., J. Lightwave Technol. 26, 8 (2008).

9. A. Laurain et al., Opt. Expr. 17, 9503-9508 (2009).

10. A. P. Bogatov, P. G. Eliseev, and B. N. Sverdlov, IEEE J. Quantum Electron. 11, 510-515 (1975).

11. G. P. Agrawal, J. Opt. Soc. Am. B 5, 147 (1988). 